\title{
Moviéndose en los márgenes: un estudio de caso sobre la identidad activista en la formación del profesorado de Música en Chile
}

\author{
Moving on the edge: a case study about activist identity in music teacher education in \\ Chile
}
Felipe-Javier Zamorano-Valenzuela felipe.zamorano.v@gmail.com Universidad de Granada Granada, España
ORCID: http://0000-0002-1309-4726

doi: 10.7203/LEEME.46.16278

Recibido: 13-12-2019 Aceptado: 28-04-2020. Contacto y correspondencia: Felipe Javier Zamorano Valenzuela. Universidad de Granada, Facultad de Ciencias de la Educación, Campus de Cartuja, s/n, C.P. 18071 Granada. España.

\section{Resumen}

El presente artículo es parte de una investigación que tiene como objetivo comprender la forma en que se construye la identidad docente en los programas de formación del profesorado de música en Chile. Se expone el caso de una titulación para ilustrar una formación basada en principios críticos y políticos, la cual permitiría la construcción de una identidad activista. En primer lugar, el artículo describe las identidades que emergen en un contexto de control sobre la profesión docente, para luego exponer algunas condiciones en la formación del profesorado para el florecimiento de la identidad activista. Se explica brevemente el planteamiento metodológico, para finalmente entregar evidencias del caso, a partir de documentos oficiales, entrevistas, grupo de discusión y observaciones. Estas evidencias permiten concluir que el territorio formativo del caso, que pone en su centro la integración curricular, investigación educativa, autonomía didáctica, la reconstrucción de la historia del profesorado y los espacios democráticos, interdisciplinares y movilizados, permitiría el florecimiento de una identidad políticamente comprometida y que más que técnica es propositiva.

Palabras clave: Formación del profesorado de música; identidad activista; educación musical; estandarización; estudio de caso.

\begin{abstract}
The paper present is part of a research project that aims to understand the way in which the teacher identity is constructed in the education of music teacher at universities in Chile. It is present the case of a study program to illustrate how a critical and political based learning allows the construction of an activist identity. First the paper describes the identities that emerge in a context of structural control of the music teacher education, and then proceeds to present some of the conditions in which the activist identity can flourish. It is explained briefly the methodological approach, and further on proceed to present the evidence obtained from the case study through document analysis, interviews, discussion groups, and observations. These evidences lead to the conclusion that the learning approach in the study program, based on the curricular integration, pedagogical research, autonomic didactic, the reconstruction of the history of teachers, and the democratic, interdisciplinary, and open spaces, allows the flourishing of a politically-engaged identity, and it has an active purposeful approach, rather than a merely technical one for teaching.
\end{abstract}

Key words: Music teacher's education; activist identity; music education; standardization; case study.

@Felipe Javier Zamorano Valenzuela. The content of this article is the sole responsibility of the authors. The Revista Electrónica de LEEME and Universitat de València are not liable for any legal actions that may arise involving the article's content. Revista Electrónica de LEEME - Lista Electrónica Europea de Música en la Educación-. http://ojs.uv.es/index/php/LEEME/index ISSN: 1575-9563. Editores: Universidad de Valencia y Jesús Tejada. Visibilidad de esta revista: SCOPUS, Emerging Sources Citation Index (Clarivate), EBSCO, CINDOC (CSIC), Citefactor, COPAC, Dialnet, DICE (CSIC), DOAJ, e-revistas (CSIC), EBSCO Premier, ERIH+, Gale Cengage Learning, INRECS, IRESIE, LATINDEX, MIAR, OCLC Worldcat, RESH, REDIB, RILM Core Journals, SUDOC, ULRICHS. Esta revista es de acceso libre mediante licencia Creative Commons 4.0 CC by. Política de archivado: etiqueta verde SHERPA-ROMEO 


\section{Introducción}

Durante los últimos años se ha instalado con fuerza la idea de un profesorado capaz de emprender y gestionar sus actuaciones tanto dentro como fuera del aula. Por lo general, son conceptos sacados del mundo empresarial que han llamado a los programas de formación del profesorado a abrazar una cultura emprendedora con el argumento de que podría convertir a los docentes en un grupo eficaz y efectivo en el cumplimiento de los objetivos propuestos (Laval, 2004). Al mismo tiempo, gracias al bajo nivel de confianza de la sociedad con sus instituciones educativas (Woodford, 2005), se han desplegado con facilidad estrategias para el control y mejora del trabajo del profesorado (Popkewitz, 1994; Whitty, 2001), en donde "la regulación, la aplicación y las sanciones son necesarias para asegurar su cumplimiento. De sus profesionales requiere un ordenamiento propio, no basado en el juicio individual o moral, sino en el cumplimiento de edictos y mandatos aplicados externamente" (Sachs, 2005, p.581). De esta manera, la docencia comienza a ser subsumida por "una tecnología, una cultura y una modalidad de reglamentación que utiliza evaluaciones, comparaciones e indicadores como medios para controlar, desgastar y producir cambio" (Ball, 2003, p.90). En otras palabras, la formación docente se ve arrinconada por políticas educativas basadas en conceptos de calidad y eficiencia, que de Perogrullo solo son capaces de lograr sus objetivos a través de la fijación de ideales formativos: estándares.

Chile, en su afán de posicionarse dentro del concepto de país moderno miembro de la OCDE, ha expuesto a su sistema educativo a esa cierta evangelización que implica la aceptación de los estándares, los que presuntamente objetivos y con referencias al sentido común, permitirían reintegrar el estatus de profesión a la docencia y la garantía de calidad a su profesorado (Cavieres-Fernández y Apple, 2016). Por ello, desde el año 2014, los programas de formación del profesorado de música (en adelante, PFPM) han debido acomodarse al nuevo marco de estándares musicales y pedagógicos, argumentando libertad en sus propuestas formativas desde la supuesta autonomía que les confiere el Ministerio de Educación. Sin embargo, la realidad es que estos estándares actúan, primero, en los procesos de acreditación a los cuales deben someterse obligatoriamente los PFPM y, segundo, en las dos evaluaciones diagnósticas del profesorado en formación. Esto revela que los estándares son "utilizados como una herramienta gerencialista para medir la eficiencia y efectividad de los sistemas, instituciones e individuos" (Sachs, 2005, p.583), y que, en términos prácticos, más que promover la formación crítica han establecido un conjunto de metas de corte técnico a las cuales responder (Assaél et al., 2011; Carrasco et al., 2019). Bajo estas condiciones, cabe preguntarse en qué lugar queda dentro de los planes de estudio una formación en donde el profesorado de música desarrolle discursos autónomos, críticos y políticos sobre problemáticas sociales y la educación musical, para así poder atender a la ineludible función de la escuela de ser inclusiva, democrática y socialmente comprometida. La eficiencia sin duda es necesaria; la formación crítica, también.

@ Felipe Javier Zamorano Valenzuela. The content of this article is the sole responsibility of the authors. The Revista Electrónica de LEEME and Universitat de València are not liable for any legal actions that may arise involving the article's content. Revista Electrónica de LEEME - Lista Electrónica Europea de Música en la Educaciónhttp://ojs.uv.es/index/php/LEEME/index ISSN: 1575-9563. Editores: Universidad de Valencia y Jesús Tejada. Visibilidad de esta revista: SCOPUS, Emerging Sources Citation Index (Clarivate), EBSCO, CINDOC (CSIC), Citefactor, COPAC, Dialnet, DICE (CSIC), DOAJ, e-revistas (CSIC), EBSCO Premier, ERIH+, Gale Cengage Learning, INRECS, IRESIE, LATINDEX, MIAR, OCLC Worldcat, RESH, REDIB, RILM Core Journals, SUDOC, ULRICHS. Esta revista es de acceso libre mediante licencia Creative Commons 4.0 CC by. Política de archivado: etiqueta verde SHERPA-ROMEO. 
Este artículo presenta un estudio de caso realizado sobre un PFPM en una universidad pública chilena con el fin de ilustrar un territorio formativo que apunta a la construcción de la identidad activista en un contexto altamente controlado y tecnificado. Para tal objetivo, se realiza, en primer lugar, una revisión de la literatura sobre las identidades que emergen en el contexto de estandarización, identidad empresarial y activista, así como ciertas condiciones para el florecimiento de esta última. En segundo lugar, se expone sucintamente el diseño metodológico, seguido de la contextualización del caso para, en tercer lugar, ofrecer descripciones e interpretaciones de este. El artículo concluye con una serie de consideraciones sumarias sobre los datos encontrados, siendo el principal resultado la posibilidad de formar para el activismo en un contexto donde el control docente en base a estándares está instalado.

\subsection{Formación del profesorado de música e identidad activista}

Chile y el mundo atraviesan por momentos en donde los problemas sociales se profundizan y radicalizan. Es fácil encontrarse con noticias respecto al auge de los movimientos migratorios y al brote de gobiernos populistas, los cuales proporcionan respuestas sencillas a problemas complejos. El currículo escolar y la formación del profesorado no puede permanecer inane a esta situación, pues los cambios sociales esperan una respuesta por parte del sistema educativo (Marcelo y Vaillant, 2018). Por tanto, cabe preguntarse qué profesorado de música está formándose, pues la probable falta de destreza política de la educación musical actual, sus argumentos y constructos teóricos (Schmidt, 2017; Woodford, 2005), condicionarían las posibilidades de lo que significa ser profesor de música (Colwell, 2018). De esta manera, se instala la duda sobre si los programas de PFPM están tendiendo a formar solo bajo los criterios de eficiencia, dejando de lado la construcción de un sujeto político y autónomo que entienda la música en la escuela como un medio de comprensión democrática para la transformación social y la promoción de nuevos discursos (Abrahams, 2005). Negar este componente crítico quizás conduzca hacia una formación que deambule entre los tecnicismos y el lenguaje práctico de la música y la pedagogía.

El dilema diario es si contribuir al mantenimiento del statu quo social o enfrentarlo (...). Esta dualidad entre conformidad social y cambio es un reflejo de la naturaleza política de la educación [musical], que ha estado, está y estará siempre en crisis, porque la educación no es una ciencia determinista sino un asunto intrínsecamente político y, por lo tanto, controvertido (Rusinek y Aróstegui, 2015, p.78).

Esta disyuntiva la recoge Sachs (2003), al reconocer dos tipos de identidades docentes que emergen en el contexto de estandarización. La primera, identidad empresarial, tiene relación con un profesionalismo gerencial, pues se vincula afirmativamente con los controles políticoeducativos y se caracteriza por ser individualista, competitiva, controlada y externamente definida. Esto significa, que se ha diseñado al "profesor en formación más como un técnico que como un profesional capaz de tener juicio crítico y reflexión. Enseñar se convierte simplemente en un empleo, en un conjunto de competencias que se debe adquirir" (Ball, 2003, p.93) para

@ Felipe Javier Zamorano Valenzuela. The content of this article is the sole responsibility of the authors. The Revista Electrónica de LEEME and Universitat de València are not liable for any legal actions that may arise involving the article's content. Revista Electrónica de LEEME - Lista Electrónica Europea de Música en la Educación-. http://ojs.uv.es/index/php/LEEME/index ISSN: 1575-9563. Editores: Universidad de Valencia y Jesús Tejada. Visibilidad de esta revista: SCOPUS, Emerging Sources Citation Index (Clarivate), EBSCO, CINDOC (CSIC), Citefactor, COPAC, Dialnet, DICE (CSIC), DOAJ, e-revistas (CSIC), EBSCO Premier, ERIH+, Gale Cengage Learning, INRECS, IRESIE, LATINDEX, MIAR, OCLC Worldcat, RESH, REDIB, RILM Core Journals, SUDOC, ULRICHS. Esta revista es de acceso libre mediante licencia Creative Commons 4.0 CC by. Política de archivado: etiqueta verde SHERPA-ROMEO. 
responder de manera neutral pero positiva (Apple, 2001) a lo establecido por el sistema educativo: reglas de la organización escolar, currículum, evaluaciones docentes; pues lo entiende como conveniente ante la categorización y los incentivos económicos (Sisto, 2011).

La segunda, identidad activista, entra en relación con el profesionalismo democrático. "El activismo tiene sus raíces en la resistencia revolucionaria hacia la transformación. Implica el aumento de la conciencia política y el análisis crítico de las situaciones sociopolíticas y fomenta respuestas organizadas a las injusticias" (Hess, 2018, p.34). Por lo tanto, este profesorado se comprende como un agente político capaz de responder hábilmente a los controles externos (Sachs, 2003), proyectando sus acciones sobre la base de los principios de confianza activa y política generativa (Giddens, 1998, citado en Sachs, 2000). La primera, asegurando relaciones colaborativas y negociadas en la comunidad y el levantamiento de las voces de todas las personas (Capel y Morant, 2019). La segunda, desarrollando proyectos alternativos a las imposiciones políticas y curriculares, entendida como "invitación a los docentes a comprometerse con la política en lugar de consentirla y ser gestionados por ella" (Schmidt, 2017, p.21). Así, esta identidad se movería en la categoría de identidades descentradas del mercado con fondo terapéutico (Bernstein, 1998), pues más que someterse a las reglas de la educación neoliberal, optaría por la colaboración profesional y elaboración discursos alternativos a las normas educativas.

Los territorios formativos en donde pudiese florecer esta identidad comprenden "la escolarización y la formación del profesorado como elementos cruciales del movimiento hacia una sociedad más justa" (Liston y Zeichner, 1997, p.51). Desde esta perspectiva, los PFPM deben ayudar a que sus estudiantes desarrollen sus propios constructos profesionales a partir de una mirada problematizadora de los principios que subyacen al trabajo docente (Giroux, 1997). Para esto, es necesario promover espacios en donde los estudiantes

\begin{abstract}
reflexionen y cuestionen los supuestos políticos, y luego desarrollen sus propias maneras de articular sus posturas políticas/profesionales (...). El sentido de profesionalismo de los educadores exige que tomen posiciones éticas. Sus responsabilidades de promover y proteger las necesidades de los estudiantes significan que, a veces, entrarán en conflictos políticos cuando los arreglos y las políticas (o la falta de ellas) perjudican a los estudiantes. Hablar simplemente es un acto político (Marshall y Anderson, 2009, p.28).
\end{abstract}

De este modo, la revisión de los principios pedagógicos debe ser articulada a las motivaciones del futuro profesorado y sus experiencias en los centros educativos, con el objetivo de, complejizar el discurso pedagógico, repensar lo que significa ser docente, imaginar escenarios alternativos y construir herramientas para actuar en estos (Allsup, 2003).

En términos prácticos, estos territorios formativos promueven la investigación e innovación a partir de una mirada comprensiva de las problemáticas del prácticum, así como el trabajo transversal de conceptos relacionados a la justicia social por medio de contenidos y metodologías de carácter democrático dentro del aula universitaria (Zeichner, 2010). Al mismo tiempo, estos programas ponen en su centro las desigualdades promovidas por la escuela y sus

@ Felipe Javier Zamorano Valenzuela. The content of this article is the sole responsibility of the authors. The Revista Electrónica de LEEME and Universitat de València are not liable for any legal actions that may arise involving the article's content. Revista Electrónica de LEEME - Lista Electrónica Europea de Música en la Educación-. http://ojs.uv.es/index/php/LEEME/index ISSN: 1575-9563. Editores: Universidad de Valencia y Jesús Tejada. Visibilidad de esta revista: SCOPUS, Emerging Sources Citation Index (Clarivate), EBSCO, CINDOC (CSIC), Citefactor, COPAC, Dialnet, DICE (CSIC), DOAJ, e-revistas (CSIC), EBSCO Premier, ERIH+, Gale Cengage Learning, INRECS, IRESIE, LATINDEX, MIAR, OCLC Worldcat, RESH, REDIB, RILM Core Journals, SUDOC, ULRICHS. Esta revista es de acceso libre mediante licencia Creative Commons $4.0 \mathrm{CC}$ by. Política de archivado: etiqueta verde SHERPA-ROMEO. 
relaciones de poder, ayudando al profesorado a: comprender críticamente la escuela y la comunidad, pero con una alta capacidad de adaptarse a ella; incluir en sus propuestas curriculares y actividades musicales a todos sus estudiantes; abordar activa y críticamente la opresión en el aula de música, las condiciones que afectan a sus estudiantes y las problemáticas externas de la escuela; contextualizar la música desde perspectivas sociohistóricas y políticas; y apoyarse mutuamente entre docentes y estudiantes para el desarrollo de iniciativas interdisciplinares (Hess, 2018).

\section{Método}

Este artículo es parte de una investigación que tiene como objetivo comprender cómo el futuro profesorado de música construye su identidad docente en dos universidades chilenas. Para esto, se utilizó el estudio de caso como enfoque, ya que "toda institución y todo programa tienen una historia que contar sobre su origen, su desarrollo y sus logros en un determinado momento. El estudio de caso documenta e interpreta la complejidad de esta experiencia en su contexto sociopolítico concreto" (Simons, 2011, p. 15). De esta manera, se buscaba recrear la experiencia de los participantes y el caso dentro de uno mismo (Bresler y Stake, 2006), a través de la comprensión de los planes de estudio, las metodologías y ambientes, y el tránsito de estudiante a docente de música.

Tabla 1. Métodos de recogida de información en el caso

\begin{tabular}{llcc}
\hline MÉTODO & FUÉLISIS \\
DOCUMENTAL & $\begin{array}{c}\text { Guías docentes, información página } \\
\text { web y otros documentos oficiales } \\
\text { (informe de autoevaluación, } \\
\text { reglamento, protocolo de entrevistas). }\end{array}$ & $\begin{array}{c}\text { Analizar la propuesta formativa del plan de } \\
\text { estudio, sus asignaturas, perfil de ingreso y } \\
\text { egreso }\end{array}$ \\
$\begin{array}{l}\text { OBSERVACIÓN } \\
\text { DE CLASES }\end{array}$ & $\begin{array}{c}\text { Asignaturas de distintos niveles y } \\
\text { regiones formativas durante 3 meses. }\end{array}$ & $\begin{array}{c}\text { Analizar las metodologías de trabajo, } \\
\text { relación entre profesorado y estudiantes, y } \\
\text { entre estudiantes. }\end{array}$ \\
ENTREVISTAS & $\begin{array}{c}7 \text { estudiantes, 2 egresados, } \\
1 \text { directivo y 6 académicos. }\end{array}$ & $\begin{array}{c}\text { Analizar experiencias musicales previas, } \\
\text { las experiencias valoradas del caso, } \\
\text { creencias sobre el rol del profesorado de } \\
\text { música. }\end{array}$ \\
GRUPO DE & 7 estudiantes. & $\begin{array}{c}\text { Analizar creencias sobre ciertos conceptos } \\
\text { relacionados a la formación dada por el } \\
\text { caso, al rol de la educación musical y el } \\
\text { profesorado de música en la escuela. }\end{array}$ \\
\hline
\end{tabular}

Fuente: Elaboración propia

Durante el diseño de la investigación se pensó que para dar una mirada profunda a los casos era necesario utilizar diversos métodos de recogida de información. En la Tabla 1 se pueden observar los métodos aplicados en el estudio de caso que expone este artículo. Finalmente, con el objetivo de garantizar ciertos compromisos éticos con los participantes, los

@ Felipe Javier Zamorano Valenzuela. The content of this article is the sole responsibility of the authors. The Revista Electrónica de LEEME and Universitat de València are not liable for any legal actions that may arise involving the article's content. Revista Electrónica de LEEME - Lista Electrónica Europea de Música en la Educación-, http://ojs.uv.es/index/php/LEEME/index ISSN: 1575-9563. Editores: Universidad de Valencia y Jesús Tejada. Visibilidad de esta revista: SCOPUS, Emerging Sources Citation Index (Clarivate), EBSCO, CINDOC (CSIC), Citefactor, COPAC, Dialnet, DICE (CSIC), DOAJ, e-revistas (CSIC), EBSCO Premier, ERIH+, Gale Cengage Learning, INRECS, IRESIE, LATINDEX, MIAR, OCLC Worldcat, RESH, REDIB, RILM Core Journals, SUDOC, ULRICHS. Esta revista es de acceso libre mediante licencia Creative Commons 4.0 CC by. Política de archivado: etiqueta verde SHERPA-ROMEO. 
procesos de aplicación de estos métodos fueron acompañados por la entrega sistemática de los respectivos consentimientos informados.

\subsection{El caso: estructura y participantes}

El caso pertenece a una universidad pública que retoma la formación de profesorado en 1994 luego que la dictadura militar la despojara de esta. El plan de estudio analizado fue producto de la innovación curricular realizada el 2014, el que al someterse al proceso de acreditación logró una de las máximas valoraciones por parte de la agencia evaluadora, lo que supone calidad y adecuación a los estándares de formación.

El plan de estudio, que conduce al grado académico de Licenciado/a en Educación con mención, se ofrece en la modalidad consecutiva, pues durante 3 semestres se encarga de la formación pedagógica de licenciados de diversas disciplinas. Este territorio curricular tiene una carga total de 90 créditos $\left(\mathrm{CTS}^{1}\right)$ que se distribuyen homogéneamente en cada semestre, y 2 regiones formativas: psicopedagógica específica y psicopedagógica general. En la región psicopedagógica específica se encuentran 3 asignaturas de Didáctica de la Música (con módulos de Evaluación e Informática Educativa), las cuales suman 36 créditos, el $40 \%$ de la carga total. Por otro lado, en la región psicopedagógica general con 54 créditos, $60 \%$ de la carga total, se observan asignaturas sobre: investigación, psicología, historia de la escuela, currículum, formación ciudadana y ética. Finalmente, sobre las 630 horas de prácticas en los centros educativos, estas se incluyen como parte de las asignaturas de Didáctica de la Música y Taller de Investigación y Práctica, con el $72.2 \%$ y $27.8 \%$ de las horas respectivamente.

Tabla 2. Titulaciones de origen y número de estudiantes

\begin{tabular}{lcc}
\hline & & $\mathrm{N}^{\circ}$ DE ESTUDIANTES \\
\hline TITULACIÓN & Teoría de la Música & 3 \\
Composición Musical & 4 \\
Ingeniería en Sonido & 1 \\
& $\begin{array}{c}\text { Interpretación en Canto, mención } \\
\text { Dirección de Coros } \\
\text { Intérprete Superior en Guitarra } \\
\text { Licenciatura en Música, mención en } \\
\text { Dirección de Orquestas }\end{array}$ & 1 \\
& & 1 \\
\hline
\end{tabular}

Fuente: Elaboración propia

\footnotetext{
${ }^{1} 1$ CTS equivale a 27 horas de trabajo.
}

@Felipe Javier Zamorano Valenzuela. The content of this article is the sole responsibility of the authors. The Revista Electrónica de LEEME and Universitat de València are not liable for any legal actions that may arise involving the article's content. Revista Electrónica de LEEME - Lista Electrónica Europea de Música en la Educación-. http://ojs.uv.es/index/php/LEEME/index ISSN: 1575-9563. Editores: Universidad de Valencia y Jesús Tejada. Visibilidad de esta revista: SCOPUS, Emerging Sources Citation Index (Clarivate), EBSCO, CINDOC (CSIC), Citefactor, COPAC, Dialnet, DICE (CSIC), DOAJ, e-revistas (CSIC), EBSCO Premier, ERIH+, Gale Cengage Learning, INRECS, IRESIE, LATINDEX, MIAR, OCLC Worldcat, RESH, REDIB, RILM Core Journals, SUDOC, ULRICHS. Esta revista es de acceso libre mediante licencia Creative Commons 4.0 CC by. Política de archivado: etiqueta verde SHERPA-ROMEO. 
Respecto a los participantes del caso, los 11 estudiantes de la especialidad de música (todos hombres) provienen de 4 instituciones, en donde han cursado estudios musicales con extensiones variadas: entre 5 y 10 años dependiendo de la titulación. En la Tabla 2, se observan las titulaciones de origen agrupadas según foco. Por otro lado, los académicos del caso poseen en su mayoría el grado doctor obtenido en el extranjero, al igual que la académica a cargo de las asignaturas de Didáctica de la Música, la cual es profesora de música y doctora en musicología.

\section{Temas de investigación}

Los principales hallazgos que se presentan a continuación son consecuencia de la interpretación durante y después de la recogida de información. Los temas de investigación se organizan desde la propuesta oficial del plan de estudio, pasando por las experiencias de los estudiantes $^{2}$ y llegando a ciertas creencias sobre el rol docente y la educación musical.

\subsection{Propuesta formativa}

El plan de estudio indica el territorio curricular por el que transita el futuro profesorado de música. De él se levantaron ideas relativas a la integración de asignaturas, diálogo entre la investigación y el prácticum, búsqueda del conocimiento didáctico, enfoque político y reconstrucción del colectivo docente.

a) Núcleos formativos: El caso promueve el desarrollo y profundización en núcleos de trabajo para cada semestre. Esto significa que, aunque cada asignatura del plan de estudio desarrolla sus contenidos específicos, se establecen

núcleos de problemas por semestre, a los que las actividades curriculares colaboran a problematizar, indagar y responder. Los núcleos de problemas por semestre son los siguientes: a) Semestre 1, "Comprensión crítica de la institución escolar"; b) Semestre 2, "Construcción de las relaciones pedagógicas"; c) Semestre 3, "Proyectos de acción en la escuela"3.

Esto permite, en primer lugar, establecer un camino formativo construido en base a los núcleos a problematizar y, en segundo lugar, realizar una integración curricular por medio de problemas comunes en todas las asignaturas.

b) La investigación docente y el prácticum: La formación de este profesorado se vuelve significativa en tanto comienzan a dar sentido a sus experiencias en las escuelas. El Taller de Investigación y Práctica se presenta como un espacio que permite vincular, a través de la investigación educativa, las biografías del estudiantado, sus

\footnotetext{
${ }^{2}$ Los nombres de los participantes han sido modificados con el objetivo de resguardar el anonimato comprometido al momento de acceder al caso.

${ }^{3}$ Información extraída del Reglamento de la Titulación.
}

@Felipe Javier Zamorano Valenzuela. The content of this article is the sole responsibility of the authors. The Revista Electrónica de LEEME and Universitat de València are not liable for any legal actions that may arise involving the article's content. Revista Electrónica de LEEME - Lista Electrónica Europea de Música en la Educación-. http://ojs.uv.es/index/php/LEEME/index ISSN: 1575-9563. Editores: Universidad de Valencia y Jesús Tejada. Visibilidad de esta revista: SCOPUS, Emerging Sources Citation Index (Clarivate), EBSCO, CINDOC (CSIC), Citefactor, COPAC, Dialnet, DICE (CSIC), DOAJ, e-revistas (CSIC), EBSCO Premier, ERIH+, Gale Cengage Learning, INRECS, IRESIE, LATINDEX, MIAR, OCLC Worldcat, RESH, REDIB, RILM Core Journals, SUDOC, ULRICHS. Esta revista es de acceso libre mediante licencia Creative Commons 4.0 CC by. Política de archivado: etiqueta verde SHERPA-ROMEO 
experiencias en los centros educativos y las reflexiones en base a los núcleos de trabajo. Sobre esto, una académica comenta que

\begin{abstract}
uno de los acuerdos curriculares para el primer semestre es que los chicos conozcan los paradigmas investigativos (...). Entonces yo me preguntaba como profesora de qué forma los chicos que traen cuatro o cinco años de licenciatura en el cuerpo y en la cabeza, van a conocer que existen modalidades y enfoques investigativos diferentes, al suyo propio. Los de ciencias con relación a los de humanidades, los de artes en relación con los demás. Y me pareció que era mucho más fructífero que ellos miraran sus propias investigaciones a partir de ciertas preguntas orientadoras que son muy básicas pero fundamentales como: ¿qué investigué?, ¿cómo lo hice?, ¿para qué investigué?, ¿qué lugar tenía yo como investigador?, ¿en la investigación pude interferir o fui un engranaje más de unas lógicas y unos propósitos donde yo no estaba involucrado?
\end{abstract}

Preguntas que buscan articular las experiencias primarias de investigación con nuevas perspectivas de indagación docente en los centros de práctica, para así construir inicialmente una narrativa personal sobre la investigación.

c) Didáctica de autor: La asignatura de Didáctica de la Música promueve la construcción personal del saber didáctico, a partir de actividades de discusión sobre los fundamentos de la educación musical, el currículum, los métodos musicales y las experiencias en los centros de práctica. Según la académica de la asignatura, se busca que los estudiantes construyan

\begin{abstract}
una didáctica de autor o una pedagogía de autor. Es como yo me identifico como profesor en este trayecto que tengo, qué para mi es importante. (...) Claro, entonces finalmente las metodologías tienen que ver con el uso de múltiples maneras como de enfrentarse, por ejemplo, a los conceptos, a los contenidos, pero también a las prácticas, como, por ejemplo, las analogías, lo inductivo y lo deductivo. Pero lo más importante es, yo diría, el empoderamiento de los propios estudiantes respecto de cómo se trabaja en la clase misma.
\end{abstract}

De este modo, se asume la necesidad de reflexionar y dotar de sentido a las decisiones didácticas que se toman, pues, aunque existe una revisión general de los métodos musicales (Orff, Kodály, Suzuki, entre otros) y de las tareas administrativas relativas al trabajo en la escuela, esta se ve minimizada por el desarrollo de una mirada crítica y autónoma del estudiantado.

d) Enfoque político y acción fundamentada: El reglamento del caso propone seis dominios formativos, entre ellos el formativo-político. Este dominio:

tiene por objetivo la formación ciudadana de los jóvenes, tanto en el desarrollo de prácticas de convivencia democrática, resolución pacífica, dialogada y argumentada de conflictos, como en el desarrollo de una conciencia y sensibilidad social respetuosas de la diversidad y la generación de una capacidad crítico-propositiva en todos sus ámbitos de acción. ${ }^{4}$

\footnotetext{
${ }^{4}$ Información extraída del Reglamento de la Titulación.
}

@ Felipe Javier Zamorano Valenzuela. The content of this article is the sole responsibility of the authors. The Revista Electrónica de LEEME and Universitat de València are not liable for any legal actions that may arise involving the article's content. Revista Electrónica de LEEME - Lista Electrónica Europea de Música en la Educaciónhttp://ojs.uv.es/index/php/LEEME/index ISSN: 1575-9563. Editores: Universidad de Valencia y Jesús Tejada. Visibilidad de esta revista: SCOPUS, Emerging Sources Citation Index (Clarivate), EBSCO, CINDOC (CSIC), Citefactor, COPAC, Dialnet, DICE (CSIC), DOAJ, e-revistas (CSIC), EBSCO Premier, ERIH+, Gale Cengage Learning, INRECS, IRESIE, LATINDEX, MIAR, OCLC Worldcat, RESH, REDIB, RILM Core Journals, SUDOC, ULRICHS. Esta revista es de acceso libre mediante licencia Creative Commons 4.0 CC by. Política de archivado: etiqueta verde SHERPA-ROMEO. 
Propuesta relacionada al desarrollo de una conciencia democrática del profesorado que reconoce a la escuela como un espacio diverso y complejo. Para esto, según un académico, la titulación busca formar

[un] profesional que sea autónomo, empoderado en lo suyo y que lo haga a partir de argumentos, no sólo de intencionalidades o pura intuición digamos. (...) Esperamos que el egresado sea un profesor autónomo en sus decisiones, que no sea sólo un aplicador pasivo de un currículo nacional porque para eso tú tienes perfiles técnicos o neoconductista de profesores. Entonces, queremos profesores autónomos que conozcan los contextos para lograr que se desarrollen de manera mucho más integral en los márgenes que la escuela de hoy permite.

De esta forma, el alumnado debe desarrollar la capacidad de moverse autónoma y estratégicamente, a través de discursos y prácticas, más que intuitivas, fundamentadas en la comprensión crítica del quehacer docente.

e) Reconstrucción del movimiento docente: El plan de estudios propone revisitar los antiguos movimientos docentes. Para esto, como punto de partida se reflexiona sobre la biografía formativa de los estudiantes, la construcción personal y social del sujeto docente y su relación histórica con la sociedad y el Estado. Parte de esto se observó en la asignatura de Compresión Histórica, en donde asisten estudiantes de distintas menciones.

[Nota asignatura Comprensión Histórica] El primer bloque ha comenzado con el tema de la Escuela Nueva. Ahora el segundo bloque aborda el "Plan de Reforma Integral de la Enseñanza Pública". Así, la profesora muestra un video sobre la historia de las Escuelas Consolidadas.

Académica: enfatizaría en que los profesores venían con una teoría pedagógica gracias a la Asociación General de Profesores, una visión de la educación pública. Desde la AGP vamos a lo que ahora es el Colegio de Profesores, funcionarios públicos que pasan al código laboral, es decir, como privados... Antes estaban sindicalizados y se denominaban trabajadores públicos, pero con la creación del Colegio de Profesores durante el año 73 pasan a ser considerados como profesionales ¿Por qué creen que en esta última etapa no hay nuevas propuestas de movimientos pedagógicos?

Estudiante Artes Visuales: Creo que tiene que ver con el individualismo.

Estudiante Historia: Quizás tiene que ver con la estructura de las escuelas y el sistema de evaluación nacional... Presión social sobre los profesores... Que no los valoran.

De esta manera la conversación sigue sobre la falta del tiempo para conversar y repensar la educación... Para realizar un trabajo colaborativo.

Académica: Nuestro desafío es ir construyendo... El generar las condiciones para la transformación de las propuestas pedagógicas a nivel nacional.

De esta forma, se ponen en perspectiva los movimientos docentes con el objetivo de que los estudiantes conozcan la biografía social de estos colectivos y, así, desarrollen cierta apropiación gremial.

@ Felipe Javier Zamorano Valenzuela. The content of this article is the sole responsibility of the authors. The Revista Electrónica de LEEME and Universitat de València are not liable for any legal actions that may arise involving the article's content. Revista Electrónica de LEEME - Lista Electrónica Europea de Música en la Educación-. http://ojs.uv.es/index/php/LEEME/index ISSN: 1575-9563. Editores: Universidad de Valencia y Jesús Tejada. Visibilidad de esta revista: SCOPUS, Emerging Sources Citation Index (Clarivate), EBSCO, CINDOC (CSIC), Citefactor, COPAC, Dialnet, DICE (CSIC), DOAJ, e-revistas (CSIC), EBSCO Premier, ERIH+, Gale Cengage Learning, INRECS, IRESIE, LATINDEX, MIAR, OCLC Worldcat, RESH, REDIB, RILM Core Journals, SUDOC, ULRICHS. Esta revista es de acceso libre mediante licencia Creative Commons 4.0 CC by. Política de archivado: etiqueta verde SHERPA-ROMEO. 


\section{2. Reformando la experiencia}

El caso se presenta como un espacio abierto que contrasta con la experiencia en la formación musical de los estudiantes. Esto significa que la formación pedagógica se vive con nuevas formas de relacionarse con el cuerpo académico, el conocimiento y las temáticas sociales.

a) Del "Conservatorio" a la Pedagogía: La formación musical les ha permitido a los estudiantes conocer la tradición academicista, lo que implica que vivan con mayor libertad el nuevo espacio pedagógico, pues se acercan a otras maneras de aprender y relacionarse con el cuerpo académico. Antonio, de primer semestre, señala:

eres barítono y tienes que cantar cosas de barítono y no puedes cantar otras cosas. Olvídalo. Hay pecados en la música, cosas que no se pueden hacer, tabús. Pero aquí pues, aquí nada es tabú. Hay un mundo mucho más abierto. Entonces, la parte de las humanidades, de poder escribir lo que siento en un texto, siempre me gustó mucho y estudiando música, en los nueve años jamás tuve esa oportunidad, pero ahora sí que siento que sí... Dar una visión crítica de algo.

En sintonía con esta idea, Alfonso, de primer semestre, apunta que:

la figura del profesor, del maestro, se ha instalado (...). Todo lo contrario de lo que he visto aquí. Ninguno de los profesores que he visto hasta ahora tiene esa actitud, como de: "yo soy el profe, si ustedes no me hacen caso, mal” ¡Eso no está! (....). No hay una actitud de prepotencia o de imposición de lo que ella piensa. Siempre hay un diálogo y esa cuestión creo que es sumamente importante para formar a futuros profesores.

De esta manera, se entiende que el alumnado se mueve desde un espacio restringido a uno abierto que le permite integrar sus voces en el proceso de construcción profesional y reconocer otras formas de relacionarse pedagógicamente.

b) De lo disciplinar a lo interdisciplinar: La modalidad consecutiva de esta titulación obliga a que estudiantes de diversas áreas compartan experiencias y conocimientos desde sus disciplinas. La siguiente nota de campo ilustra, brevemente, este carácter:

[Nota asignatura Taller de Investigación y Práctica] Luego de una discusión sobre la investigación de corte positivista, un grupo de estudiantes de distintas disciplinas proponen una actividad a partir de un texto de José Contreras Domingo "Estudiantes que investigan, un camino a la libertad".

Antonio (Estudiante de Música): El texto era super personal. La compañera de artes visuales nos sugirió que nos centráramos en la construcción del conocimiento.

Estudiante de Artes: La idea es que cada grupo cree una problemática diferente y luego la intercambiamos.

Antonio: Quizás es tan desconocido lo que se pregunta, pero no puede quedar en blanco.

@ Felipe Javier Zamorano Valenzuela. The content of this article is the sole responsibility of the authors. The Revista Electrónica de LEEME and Universitat de València are not liable for any legal actions that may arise involving the article's content. Revista Electrónica de LEEME - Lista Electrónica Europea de Música en la Educación-, http://ojs.uv.es/index/php/LEEME/index ISSN: 1575-9563. Editores: Universidad de Valencia y Jesús Tejada. Visibilidad de esta revista: SCOPUS, Emerging Sources Citation Index (Clarivate), EBSCO, CINDOC (CSIC), Citefactor, COPAC, Dialnet, DICE (CSIC), DOAJ, e-revistas (CSIC), EBSCO Premier, ERIH+, Gale Cengage Learning, INRECS, IRESIE, LATINDEX, MIAR, OCLC Worldcat, RESH, REDIB, RILM Core Journals, SUDOC, ULRICHS. Esta revista es de acceso libre mediante licencia Creative Commons 4.0 CC by. Política de archivado: etiqueta verde SHERPA-ROMEO. 


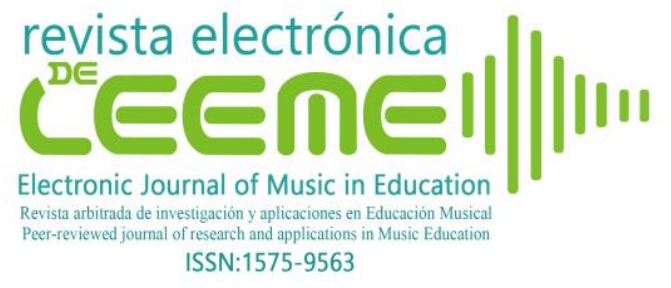

Felipe Javier Zamorano Valenzuela

Moviéndose en los márgenes: un estudio de caso sobre la identidad activista en la formación del profesorado de Música en Chile (Revista Electrónica de LEEME) Número 46, pp. 127-142 https://ojs.uv.es/index.php/LEEME/index ISSN:1575-9563

Un grupo plantea realizar una representación visual a partir de una melodía (...). Luego de que cada grupo intentara responder a la problemática planteada, algunos estudiantes dan sus apreciaciones sobre el ejercicio.

Jorge (Estudiante de Música): Bueno, como hace tiempo que no lo veíamos [algunos contenidos] fue complejo. Esa era la gracia de la actividad... Siempre vamos a estar expuestos a estas situaciones.

Estudiante de Artes: En la creación de la actividad nosotros pensamos en la pedagogía de lo singular [que tomaba el texto] y en el verse y decirse en forma grupal, creando un ejercicio y resolviendo desde otra disciplina.

De este modo, se ponen en juego diversas perspectivas disciplinares que, probablemente, permitan pensar integradamente el currículum escolar, acercarse a otras asignaturas y promover la colaboración entre el futuro profesorado.

c) La movilización social como espacio de aprendizaje: El compromiso de los estudiantes y los académicos con las movilizaciones sociales demuestra la apertura del caso a las problemáticas que ellas plantean.

[Nota Comienzo de movilizaciones] Ya terminado el primer mes de observación, comienza un movimiento de protesta dentro de la universidad. Se trata del movimiento feminista que reclama sobre casos de acoso sexual dentro de las universidades. Gracias a un estudiante pudimos acceder a uno de los calendarios semanales de actividades durante la paralización académica.

Actividades de reflexión y protesta (Tabla 3) que se extendieron por lo menos un mes más y en donde se pudo observar también la participación de los académicos. En este sentido, una de las académicas entrevistadas comenta el valor de la movilización como espacio formativo:

\begin{abstract}
a pesar de nuestras condiciones materiales, nosotros tenemos estudiantes que en general se preguntan sobre el sentido de las cosas. Se comprometen más menos, porque eso también es una cuestión individual, no es una orden, sino que es una cuestión de convicción personal. Lo hacen desde sus lugares particulares, y eso me alegra profundamente por lo que estamos haciendo aquí. De lo que estamos acompañando (...). O sea, nosotros estamos atendiendo a las cuestiones que nos plantean nuestras estudiantes, y nos preocupa que ellas y ellos, también ellos, tengan lugares seguros para formarse y también que puedan desarrollar y fortalecer una dimensión ética del trabajo, porque nuestro trabajo es de mucho cuidado.
\end{abstract}

De esta suerte, se entiende que el alumnado tiene un especial interés en abordar activamente las problemáticas sociales, así como el cuerpo académico en comprometerse en el acompañamiento de los procesos de movilización, comprendiendo que estos también son espacios de formación docente.

@Felipe Javier Zamorano Valenzuela. The content of this article is the sole responsibility of the authors. The Revista Electrónica de LEEME and Universitat de València are not liable for any legal actions that may arise involving the article's content. Revista Electrónica de LEEME - Lista Electrónica Europea de Música en la Educación-. http://ojs.uv.es/index/php/LEEME/index ISSN: 1575-9563. Editores: Universidad de Valencia y Jesús Tejada. Visibilidad de esta revista: SCOPUS, Emerging Sources Citation Index (Clarivate), EBSCO, CINDOC (CSIC), Citefactor, COPAC, Dialnet, DICE (CSIC), DOAJ, e-revistas (CSIC), EBSCO Premier, ERIH+, Gale Cengage Learning, INRECS, IRESIE, LATINDEX, MIAR, OCLC Worldcat, RESH, REDIB, RILM Core Journals, SUDOC, ULRICHS. Esta revista es de acceso libre mediante licencia Creative Commons 4.0 CC by. Política de archivado: etiqueta verde SHERPA-ROMEO. 
Tabla 3. Cronograma de actividades durante segunda semana de movilizaciones

\begin{tabular}{|c|c|c|}
\hline MIÉRCOLES 9 & JUEVES 10 & VIERNES 11 \\
\hline $\begin{array}{c}\text { "Posible almuerzo" } \\
\text { Hora: 13:00, ágora de Filosofía. } \\
\text { (Se está organizando una posible } \\
\text { lentejada. Se avisará) }\end{array}$ & $\begin{array}{c}\text { Asamblea de mujeres (Filosofía y } \\
\text { Humanidades) } \\
\text { Hora: 10:00, en la Facultad. }\end{array}$ & $\begin{array}{c}\text { Asamblea de mujeres, } \\
\text { triestamental (Filosofía) } \\
\text { Hora: 10:00, en la Facultad. }\end{array}$ \\
\hline $\begin{array}{c}\text { Asamblea } \\
\text { Hora: 14:30, sala por confirmar } \\
\text { (Filosofía). (Tabla: determinar } \\
\text { orgánica de Generación ante } \\
\text { movilizaciones, un protocolo } \\
\text { contra acoso, y reunión viernes) }\end{array}$ & $\begin{array}{c}\text { Jornada reflexiva sobre género y } \\
\text { educación sexista en contexto escolar } \\
\text { Hora: 15:00, sala por confirmar } \\
\text { (Filosofía). (Actividad extendida a } \\
\text { estudiantes y profesores, y público en } \\
\text { general) }\end{array}$ & $\begin{array}{c}\text { Junta reflexiva y recreativa para } \\
\text { conocernos G-25 } \\
\text { Hora: 13:00, lugar por confirmar. }\end{array}$ \\
\hline $\begin{array}{c}\text { Asamblea extraordinaria de } \\
\text { mujeres } \\
\text { Hora: } 17: 00 \text {, sala por confirmar } \\
\text { (Filosofía) }\end{array}$ & & $\begin{array}{c}\text { Marcha "Ni una menos" } \\
\text { Hora: 18:30. Plaza Italia, juntándose } \\
\text { en la Facultad de Derecho, desde } \\
17: 30\end{array}$ \\
\hline $\begin{array}{l}\text { Taller para el cuidado de la voz } \\
\text { Hora: post asamblea (Antonio } \\
\text { propuso esta actividad. Se hará en } \\
\text { dos bloques seguidos, ya que hay } \\
\text { asamblea extraordinaria de } \\
\text { mujeres a la misma hora) }\end{array}$ & & \\
\hline
\end{tabular}

Fuente: Estudiante entrevistado

\subsection{El rol del profesorado de Música}

El plan de estudio y sus relaciones cotidianas marcan ciertas creencias sobre el rol docente y la educación musical. Estas, orientadas principalmente hacia posturas reflexivas, críticas y políticas, son reconocidas por los estudiantes del caso. Como consecuencia de lo anterior, Cristian, de tercer semestre, señala:

el rol pedagógico es político ¡es político! Yo creo que no debe caber duda al respecto. Y hay que ser agente político transformador en el sentido de transformar la escuela en un ente crítico, un ente crítico desde lo estudiantes, un ente crítico desde los mismos profesores, desde los funcionarios, porque actualmente no lo está siendo, está siendo meramente una institución reproductora del status quo (...). Entonces yo creo que como profesores tenemos que hacernos cargo del análisis, de la crítica, y de motivar la reflexión, y también si se puede, motivar y motivarse en las distintas instancias, en los distintos movimientos que están surgiendo actualmente. Creo que como profesores debemos tener una postura firme al respecto. Por ejemplo, esto de la educación no sexista, educación feminista (...). El colectivo de profesores debería tener una postura al respecto, igual que los colectivos de trabajadores, igual que muchos entes importantes dentro de la sociedad (...). Pero no solo quedar en la postura, (...) ir más allá siempre. Y si somos actores políticos como profesores tenemos que hacernos cargo y tomar la sartén por el mango al respecto. 
Desde esta perspectiva, se entiende que el docente debe comprometerse, crítica y colectivamente, con el objetivo de transformar la escuela. A esta idea, Daniel, uno de los egresados del caso, explica que los focos de su trabajo como docente son:

enseñarles a los chicos un punto de partida con lo que dice el programa, darle una base musical sólida, a través ojalá de herramientas, o sea, a través de elementos que a ellos les hagan sentido. Por ejemplo, cantar canciones ya del "1800", que son súper valorables y todo, pero a los niños de hoy en día no les hacen sentido. (...) Acercarlos a la música de una forma amigable, atractiva, respetuosa. Y también está la otra dimensión que es lo social, que veces, se pone cuesta arriba igual, sobre todo en los profesores que tenemos pocas horas con los cursos, intentar llegar más allá de la música, llegar al pensamiento crítico, a la reflexión de lo que está ocurriendo hoy en día en las calles.

De este modo, el discurso del egresado se ve envuelto por un fuerte sentido de realidad de lo que pasa en la escuela y el aula de música. Sin embargo, este es capaz de comprender la educación musical como un espacio para el desarrollo del pensamiento crítico y la integración de las problemáticas socioculturales que afectan a la escuela.

\section{Discusión y conclusiones}

Las evidencias expuestas permiten concluir que el caso es un territorio formativo fértil en donde puede fácilmente florecer la identidad activista, pues el intenso plan de estudio es capaz de articular asignaturas y procesos de formación sin límites fuertes, ayudado por los núcleos de trabajo, las biografías de sus estudiantes y la investigación educativa con relación al prácticum. Así también, se promueve la autonomía de los estudiantes a través de la construcción de una "didáctica de autor" y distancia de los métodos musicales, lo que facilita el desarrollo de una mirada pedagógica, crítica y problematizadora, que integra temas socioeducativos generales y musicales. Lo anterior, se ve apoyado por la reconstrucción de los movimientos docentes en su contexto social, la cual da la posibilidad de conocer y valorar el trabajo colectivo del profesorado. Algo así como un ejercicio de memoria del trabajo docente que puede ser proyectado colectivamente al futuro.

Además, el caso permite a los estudiantes convertirse en docentes de música en espacios interdisciplinares, con relaciones de carácter horizontal y permeados por las demandas sociales. De esta manera, el caso se vive como una antítesis de la formación musical de origen, pues se ha pasado de un espacio de relaciones verticales y juicios objetivos sobre la música, a un territorio subjetivo e incierto, y altamente democrático. Esto amplía las perspectivas sobre la educación musical y las posibilidades de acción pedagógica, todas ellas basadas en juicios personales y colectivos.

Estudiantes y egresados reconocen en el programa un territorio con enfoque reflexivo, crítico y político, en donde la búsqueda personal es clave para el desarrollo de un profesorado autónomo y proactivo. De esta manera, la idea "educación como acto político" se asume como parte del discurso de este profesorado de música: se asignan el rol de agente transformador que

@ Felipe Javier Zamorano Valenzuela. The content of this article is the sole responsibility of the authors. The Revista Electrónica de LEEME and Universitat de València are not liable for any legal actions that may arise involving the article's content. Revista Electrónica de LEEME - Lista Electrónica Europea de Música en la Educaciónhttp://ojs.uv.es/index/php/LEEME/index ISSN: 1575-9563. Editores: Universidad de Valencia y Jesús Tejada. Visibilidad de esta revista: SCOPUS, Emerging Sources Citation Index (Clarivate), EBSCO, CINDOC (CSIC), Citefactor, COPAC, Dialnet, DICE (CSIC), DOAJ, e-revistas (CSIC), EBSCO Premier, ERIH+, Gale Cengage Learning, INRECS, IRESIE, LATINDEX, MIAR, OCLC Worldcat, RESH, REDIB, RILM Core Journals, SUDOC, ULRICHS. Esta revista es de acceso libre mediante licencia Creative Commons 4.0 CC by. Política de archivado: etiqueta verde SHERPA-ROMEO. 
debe trabajar para cambiar la realidad de la escuela chilena; creen que la enseñanza de la música debe desarrollar miradas críticas y reflexivas sobre las problemáticas sociales, sin dejar de lado experiencias musicales.

Finalmente, se entiende que el caso valiéndose de la supuesta autonomía que le confiere el marco de estándares, es intrínsecamente hábil al momento de responder con una propuesta crítica y política, probablemente marginal, en el espacio de los PFPM. La duda cabe en cómo este profesorado crítico y autónomo con ideales democráticos se moverá en su futuro trabajo docente. La escuela es un espacio complejo para el desenvolvimiento de este tipo de identidad y profesionalismo, puesto que es allí donde se intensifican y aplican directamente una serie de exámenes al profesorado. Por tanto, el reto para los PFPM de corte crítico es que su profesorado aprenda tanto a moverse estratégicamente ante las normas que rigidizan y dividen el actuar del colectivo docente, como a fundamentar sus acciones educativas y musicales con un discurso político sólido, para que el enfrentamiento sea real, no un mero activismo sin fundamentos y consecuencias.

\section{Financiación y agradecimientos}

La investigación doctoral de la que es parte este artículo ha sido financiada por el Fondo para el Fomento de la Música Nacional, línea Formación y Becas, Proyecto № 443508, Convocatoria 2018 (Ministerio de las Culturas, las Artes y el Patrimonio, Chile).

\section{Referencias}

Abrahams, F. (2005). The Application of Critical Pedagogy to Music Teaching and Learning: A Literature Review. Update: Applications of Research in Music Education, 23(2), 12-22. doi:10.1177/87551233050230020103

Allsup, R.E. (2003). Praxis and the Possible: Thoughts on the Writings of Maxine Greene and Paulo Freire. Philosophy of Music Education Review, 11(2), 157-169. Recuperado de https://www.jstor.org/stable/40327208

Apple, M.W. (2001). Markets, Standards, Teaching and Teacher Education. Journal of Teacher Education, 52(3), 182-196. doi:10.1177/0022487101052003002

Assaél, J., Cornejo, R., González, J., Redondo, J., Sánchez, R. y Sobarzo, M. (2011). La empresa educativa chilena. Educação \& Sociedade, 32(115), 305-322. doi:10.1590/S0101-73302011000200004

Ball, S.J. (2003). Profesionalismo, gerencialismo y performatividad. Revista de Educación y Pedagogía, XV(37), 87-104. Recuperado de: https://dialnet.unirioja.es/

@Felipe Javier Zamorano Valenzuela. The content of this article is the sole responsibility of the authors. The Revista Electrónica de LEEME and Universitat de València are not liable for any legal actions that may arise involving the article's content. Revista Electrónica de LEEME - Lista Electrónica Europea de Música en la Educaciónhttp://ojs.uv.es/index/php/LEEME/index ISSN: 1575-9563. Editores: Universidad de Valencia y Jesús Tejada. Visibilidad de esta revista: SCOPUS, Emerging Sources Citation Index (Clarivate), EBSCO, CINDOC (CSIC), Citefactor, COPAC, Dialnet, DICE (CSIC), DOAJ, e-revistas (CSIC), EBSCO Premier, ERIH+, Gale Cengage Learning, INRECS, IRESIE, LATINDEX, MIAR, OCLC Worldcat, RESH, REDIB, RILM Core Journals, SUDOC, ULRICHS. Esta revista es de acceso libre mediante licencia Creative Commons 4.0 CC by. Política de archivado: etiqueta verde SHERPA-ROMEO. 
Bernstein, B. (1998). Pedagogía, control simbólico e identidad: teoría, investigación y crítica. Madrid: Ediciones Morata.

Bresler, L. y Stake, R. (2006). Qualitative Research Methodology in Music Education. En R. Colwell (Ed.), MENC handbook of research methodologies (pp.270-311). Oxford: Oxford University Press.

Carrasco, C., Luzón, A., y López, V. (2019). Identidad docente y políticas de accountability: el caso de Chile. Estudios Pedagógicos, 45(2), 121-139. doi:10.4067/s071807052019000200121

Cavieres-Fernández, E. y Apple, M.W. (2016). La ley docente y la clase media: controlando el desarrollo de los profesores chilenos. Cadernos CEDES, 36(100), 265-280. doi:10.1590/cc0101-32622016171391

Capel, M. y Morant, R. (2019). Características organizativas y didácticas del concierto didáctico. Un estudio de caso sobre la coordinación entre profesorado de Música de Educación Primaria y Secundaria en la ciudad de Manises. Revista Electrónica de LEEME, 44, 63-84. doi:10.7203/LEEME.44.15868

Colwell, R. (2018). Policy and assessment. Arts Education Policy Review, 120(3), 126-139. doi:10.1080/10632913.2018.1533502

Giroux, H. (1997). Los profesores como intelectuales: hacia una pedagogía crítica del aprendizaje. Barcelona: Paidós.

Hess, J. (2018). Revolutionary Activism in Striated Spaces? Considering an Activist Music Education in K-12 Schooling. Action, Criticism \& Theory for Music Education, 17(2), 22-49. doi:10.22176/act17.2.21

Laval, C. (2004). La escuela no es una empresa: el ataque neoliberal a la enseñanza pública. Barcelona: Paidós.

Liston, D. y Zeichner, K. (1997). Formación del profesorado y condiciones sociales de la escolarización ( $2^{\circ}$ ed.). Madrid: Ediciones Morata.

Marcelo, C. y Vaillant, D. (2018). La formación inicial docente: problemas complejos respuestas disruptivas. Cuadernos de Pedagogía, 489, 27-32. Recuperado: https://www.researchgate.net/publication/325909333_La_formacion_inicial_doc

Marshall, C. y Anderson, A.L. (2009). Is it Possible to Be an Activist Educator? En C. Marshall y A.L. Anderson (Eds.), Activist educators. Breaking past limits (pp.1-30). New York: Routledge.

@ Felipe Javier Zamorano Valenzuela. The content of this article is the sole responsibility of the authors. The Revista Electrónica de LEEME and Universitat de València are not liable for any legal actions that may arise involving the article's content. Revista Electrónica de LEEME - Lista Electrónica Europea de Música en la Educación-, http://ojs.uv.es/index/php/LEEME/index ISSN: 1575-9563. Editores: Universidad de Valencia y Jesús Tejada. Visibilidad de esta revista: SCOPUS, Emerging Sources Citation Index (Clarivate), EBSCO, CINDOC (CSIC), Citefactor, COPAC, Dialnet, DICE (CSIC), DOAJ, e-revistas (CSIC), EBSCO Premier, ERIH+, Gale Cengage Learning, INRECS, IRESIE, LATINDEX, MIAR, OCLC Worldcat, RESH, REDIB, RILM Core Journals, SUDOC, ULRICHS. Esta revista es de acceso libre mediante licencia Creative Commons 4.0 CC by. Política de archivado: etiqueta verde SHERPA-ROMEO. 
Popkewitz, T.S. (1994). Política, conocimiento y poder: algunas cuestiones para el estudio de las reformas educativas. Revista de Educación, 305, 103-137. Recuperado de: https://sede.educacion.gob.es/publiventa/detalle.action?cod=493

Rusinek, G. y Aróstegui, J.L. (2015). Educational Policy Reforms and the Politics of Music Teacher Education. En C. Benedict, P. Schmidt, G. Spruce, y P. Woodford (Eds.), The Oxford Handbook of Social Justice in Music Education (pp.78-91). New York: Oxford University Press.

Sachs, J. (2000). The activist professional. Journal of Education Change, 1, 77-95. doi:10.1023/a:1010092014264

Sachs, J. (2003). The Activist Teaching Profession. Buckingham: Open University Press.

Sachs, J. (2005). Teacher Professional Standards: a Policy Strategy to Control, Regulate or Enhance the Teaching Profession? En N. Bascia, A. Cumming, A. Datnow, K. Leithwood, y D. Livingstone (Eds.), International Handbook of Educational Policy (pp.579-592). Dordrecht: Springer.

Schmidt, P. (2017). Why Policy Matters. Developing a Policy Vocabulary within Music Education. En P. Schmidt y R. Colwell (Eds.), Policy and the Political Life of Music Education (pp.11-36). New York: Oxford University Press.

Simons, H. (2011). El estudio de caso: Teoría y práctica. Madrid: Ediciones Morata.

Sisto, V. (2011). Nuevo profesionalismo y profesores: una reflexión a partir del análisis de las actuales políticas de «profesionalización» para la educación en Chile. Signo y Pensamiento, 31(59), 178-192. Recuperado de: https://www.redalyc.org/pdf/860/86022458013.pdf

Whitty, G. (2001). Teoría social y política educativa: ensayos de sociología y política de la educación. Valencia: Pomares.

Woodford, P.G. (2005). Democracy and Music Education: Liberalism, Ethics, and the Politics of Practice. Bloomington: Indiana University Press.

Zeichner, K. (2010). La formación del profesorado y la lucha por la justicia social. Madrid: Ediciones Morata.

@ Felipe Javier Zamorano Valenzuela. The content of this article is the sole responsibility of the authors. The Revista Electrónica de LEEME and Universitat de València are not liable for any legal actions that may arise involving the article's content. Revista Electrónica de LEEME - Lista Electrónica Europea de Música en la Educación-. http://ojs.uv.es/index/php/LEEME/index ISSN: 1575-9563. Editores: Universidad de Valencia y Jesús Tejada. Visibilidad de esta revista: SCOPUS, Emerging Sources Citation Index (Clarivate), EBSCO, CINDOC (CSIC), Citefactor, COPAC, Dialnet, DICE (CSIC), DOAJ, e-revistas (CSIC), EBSCO Premier, ERIH+, Gale Cengage Learning, INRECS, IRESIE, LATINDEX, MIAR, OCLC Worldcat, RESH, REDIB, RILM Core Journals, SUDOC, ULRICHS. Esta revista es de acceso libre mediante licencia Creative Commons 4.0 CC by. Política de archivado: etiqueta verde SHERPA-ROMEO. 